\title{
The Prevalence and Management of Anti-Neutrophil Cytoplasmic Antibody-Associated Vasculitis in China
}

\author{
Zhi-Ying Li ${ }^{\mathrm{a}-\mathrm{e}}$ Tian-Tian Ma ${ }^{\mathrm{a}-\mathrm{e}} \quad$ Min Chen $^{\mathrm{a}-\mathrm{e}}$ Ming-Hui Zhao ${ }^{\mathrm{a}-\mathrm{e}}$ \\ ${ }^{a}$ Renal Division, Department of Medicine, Peking University First Hospital, b Peking University Institute of \\ Nephrology, ${ }^{C}$ Peking-Tsinghua Center for Life Sciences, ${ }^{d}$ Key Laboratory of Renal Disease, Ministry of Health of \\ China, and ${ }^{\mathrm{C}}$ Key Laboratory of Chronic Kidney Disease Prevention and Treatment (Peking University), Ministry of \\ Education, Beijing, China
}

\section{Key Words}

Anti-neutrophil cytoplasmic antibody-associated vasculitis . Chinese patients $\cdot$ Management $\cdot$ Outcome $\cdot$ Prevalence

\begin{abstract}
Background: Anti-neutrophil cytoplasmic antibody (ANCA)associated vasculitis (AAV) comprises microscopic polyangiitis (MPA), granulomatosis with polyangiitis (GPA) and eosinophilic GPA (EGPA). Myeloperoxidase (MPO) and proteinase 3 (PR3) are the main antigens for ANCA. AAV is a common multisystem autoimmune disease and most of the studies on AAV have been conducted in Western countries. Nowadays in China many efforts are made to investigate this disease. Summary: This review highlights the progress in the prevalence, management and outcomes of AAV in Chinese patients. With respect to the prevalence of $A A V$, though there are no precise data, AAV is not rare in the Chinese population. In Chinese patients with AAV there is a striking preponderance of MPA, and MPO-ANCA is much more common than PR3-ANCA. Even in patients with GPA there is a predominance of MPO-ANCA over PR3-ANCA. Propylthiouracilinduced AAV and ANCA-negative pauci-immune glomerulonephritis are stated in this review as well. With respect to the management of AAV, glucocorticoids in combination with cyclophosphamide remain the mainstay of induction thera-
\end{abstract}

py. Besides, we describe predictors of different outcomes in Chinese patients, including mortality, relapse, treatment resistance and end-stage renal disease. Key Messages: AAV is not rare in the Chinese population. The disease spectrum and subtypes of ANCA are different between patients with AAV in China and Western countries. The treatment strategy for AAV in China is in consistency with that in Western countries. Predictors of different clinical outcomes are provided. Facts from East and West: Treatment options for AAV are shared between the East and West, with corticosteroid combined with cyclophosphamide being the standard regimen for inductive therapy and switching to azathioprine after remission. The major cause of death in treated patients is infection related to immunosuppressive therapy within the first year after diagnosis, and this rate might be higher in China than in Western countries. Western studies demonstrated the efficacy and safety of rituximab for induction of remission in cases with relatively mild disease and maintenance therapy, but this agent is rarely used in China.

(C) 2015 S. Karger AG, Basel

Z.-Y. Li and T.-T. Ma contributed equally to this paper.

For the prevalence and management of pauci-immune glomerulonephritis and vasculitis in Western countries, see Lionaki and Boletis, Kidney Dis 2015;1:224-234.

\section{KARGER 125\%}

(c) 2015 S. Karger AG, Basel

$2296-9381 / 15 / 0014-0216 \$ 39.50 / 0$

E-Mail karger@karger.com

www.karger.com/kdd
Min Chen, $\mathrm{MD}, \mathrm{PhD}$

Renal Division, Peking University First Hospital

8 Xishiku Street, Xicheng District

Beijing 100034 (China)

E-Mail chenmin74@sina.com 


\section{Introduction}

Vasculitis is an inflammatory process of the blood vessel wall. In the 2012 Chapel Hill Consensus Conference nomenclature of vasculitis, the classification is based on the size of the vessels involved, on the histopathology of the lesions and on clinical manifestations (table 1) [1]. Anti-neutrophil cytoplasmic antibody (ANCA)-associated vasculitis (AAV) is a small-vessel vasculitis characterized by necrotizing inflammation of small vessels (including small arteries, arterioles, capillaries and venules) in conjunction with ANCA. AAV comprises microscopic polyangiitis (MPA), granulomatosis with polyangiitis (GPA, previously Wegener's granulomatosis) and eosinophilic GPA (EGPA, previously Churg-Strauss syndrome) [1]. AAV is a group of multisystem autoimmune diseases and the most commonly involved organs are the kidneys and lungs [2].

ANCA are serological hallmarks for AAV. ANCA are predominantly IgG class autoantibodies directed against constituents of granules of neutrophils and lysosomes of monocytes. By indirect immunofluorescence (IIF) on ethanol-fixed neutrophils, two fluorescence patterns of ANCA are distinguished: the cytoplasmic staining pattern (cANCA) and the perinuclear staining pattern (pANCA). Most patients with a cANCA pattern obtained by IIF have ANCA directed against proteinase 3 (PR3), as determined by antigen-specific ELISA. Patients with pANCA mostly have ANCA directed against one of a variety of antigens, but in primary small-vessel vasculitis, the target antigen is almost invariably myeloperoxidase (MPO). The combinations of a cANCA pattern by IIF with PR3-ANCA by ELISA and a pANCA pattern by IIF with MPO-ANCA by ELISA are very specific for AAV [3]. In Western countries, PR3-ANCA are most frequent in patients with GPA and MPO-ANCA in patients with MPA, respectively [2], but levels of circulating ANCA do not always reflect disease activity [4].

\section{Prevalence of AAV in China}

AAV is one of the most common autoimmune diseases in Caucasians. Most of the studies on its prevalence have been conducted in Europe, the USA, Australia and Japan in a secondary care setting. It was reported that in European countries the overall annual incidence was approximately $10-20$ per million and the peak age of onset was 65-74 years [5]. The annual incidence of GPA is $10-12$ per million and the annual incidence of
Table 1. Classification of vasculitis according to the 2012 Chapel Hill Consensus Conference nomenclature of vasculitis

Large-vessel vasculitis

Takayasu arteritis

Giant cell arteritis

Medium-vessel vasculitis

Polyarteritis nodosa

Kawasaki disease

Small-vessel vasculitis

AAV

MPA

GPA (Wegener's granulomatosis)

EGPA (Churg-Strauss syndrome)

Immune complex small-vessel vasculitis

Anti-glomerular basement membrane disease

Cryoglobulinemic vasculitis

IgA vasculitis (Henoch-Schönlein purpura)

Hypocomplementemic urticarial vasculitis (anti-C1q vasculitis)

Variable-vessel vasculitis

Behçet's disease

Cogan's syndrome

Single-organ vasculitis

Cutaneous leukocytoclastic angiitis

Cutaneous arteritis

Primary central nervous system vasculitis

Isolated aortitis

Others

Vasculitis associated with systemic disease

Lupus vasculitis

Rheumatoid vasculitis

Sarcoid vasculitis

Others

Vasculitis associated with probable etiology

Hepatitis C virus-associated cryoglobulinemic vasculitis

Hepatitis B virus-associated vasculitis

Syphilis-associated aortitis

Drug-associated immune complex vasculitis

Drug-associated AAV

Cancer-associated vasculitis

Others

MPA is 3-10 per million in Northern Europe, while in Southern Europe MPA appears to be more frequent than GPA. EGPA is much less common than GPA or MPA, with an annual incidence of $0.5-2.0$ per million [6]. In the Japanese population, MPA is much more frequent than GPA, but the overall annual incidence of AAV is similar to that in European populations [6]. Systemic vasculitis has been reported to be associated with exposure to silica particulate $[6,7]$. Infections and ge- 
netic backgrounds are also suggested to be possible etiological factors [6].

The precise incidence of AAV in the Chinese population is still lacking. It was reported that in consistency with studies from Western countries, AAV could affect people of all ages, with elderly people being more susceptible, and the kidneys and lungs were the most vulnerable organs $[8,9]$. In our referral diagnostic center in Peking University First Hospital, over 200 new patients with AAV are diagnosed annually; this indicates that AAV is not rare in Chinese [10]. In 2014, Zhu et al. [11] analyzed the renal disease spectrum of 430 elderly patients $(\geq 65$ years) who had received renal biopsy between January 2003 and December 2012. Secondary glomerular diseases were diagnosed in 157 patients (36.5\%), and ANCA-associated glomerulonephritis (44.0\%) was the leading cause of the secondary glomerular diseases. In patients with acute kidney injury (19.5\%), the leading cause was AAV (48.1\%). These data indicate that AAV is relatively common in secondary glomerular diseases and a common cause of elderly Chinese patients with biopsy-proven acute kidney injury, but we could not exclude a selection bias since it was a single-center study.

Regarding the disease spectrum, there is a striking preponderance of MPA, constituting about $80 \%$ of patients with AAV [8]. This is in contrast with many Caucasian populations, especially in Northern Europe at high latitudes, where GPA is much more common. Accordingly MPO, rather than PR3, is the major target ANCA antigen in Chinese patients [12], even in patients with a clinical picture of GPA, $60 \%$ of them with ANCA specificity for MPO $[13,14]$. Compared with patients with 'classical' PR3-ANCA-positive GPA, the prevalence of ophthalmic and ear involvement was significantly lower, and the prevalence of elevated initial serum creatinine was significantly higher in patients with MPO-ANCA-positive GPA [13]. In renal histology, chronic lesions were more abundantly present in patients with MPO-ANCA-positive GPA than in those with PR3-ANCA-positive GPA [14]. Our further investigation suggested that MPOANCA IgG4 subclass might play a role in the development of MPO-ANCA-positive GPA [15]. The preponderance of MPO-ANCA and MPA is an epidemiological characteristic of Chinese patients with AAV. Whether these differences are caused by genetic or environmental factors is not clear.

Although individuals at all ages can be affected, over $40 \%$ of our patients were older people [16]. Compared with younger patients, older patients were more likely to suffer from MPA than GPA [16]. It was found that pul- monary involvement was more prevalent and more severe in older patients than in younger ones $[9,16]$. Those findings indicated that patients with different ages might have different susceptibility to variant disease entities and organ involvement of AAV.

Genetic factors might contribute to the development of AAV, as mentioned above. In Chinese patients several studies have suggested associations between AAV and different HLA loci. HLA-DRB1*1454 was significantly less prevalent than in normal controls, whereas DRB $1 * 1101$ was significantly more frequent in patients with MPO-ANCA-positive MPA. In patients with PR3ANCA, DRB1*1202 was more prevalent than in normal controls [17]. HLA-DPB1 variant rs3117242 was found to be associated with GPA in the Han Chinese population [18].

A recent development in $\mathrm{AAV}$ is the increasing recognition that certain drugs could induce AAV. Among these drugs, the most often implicated one is propylthiouracil (PTU), a widely used anti-thyroid drug in China [19]. Though $15-64 \%$ of patients receiving PTU have serum-positive ANCA [20], our early screening found that only one fourth of patients with positive ANCA had clinical vasculitis [19]. We also found that there were antibodies against multiple ANCA-specific antigens, especially the antigens rather than MPO and PR3 in PTU-induced ANCA. Patients with antibodies against more ANCA-specific antigens might be at increased risk of developing overt clinical vasculitis [21]. Patients with PTU-induced AAV have less organ involvement and milder lesions on renal histology compared to those with primary AAV $[22,23]$. Compared with MPOANCA in primary AAV, MPO-ANCA in PTU-induced AAV shows lack of IgG3 subclass as well as lower affinity and recognizes restricted epitopes on the MPO molecule $[15,24]$.

The histopathological hallmark of ANCA-associated glomerulonephritis is pauci-immune crescentic glomerulonephritis (CrGN). In the majority of patients with pauci-immune CrGN, the renal disorder is regarded as part of AAV. The majority of patients with pauci-immune $\mathrm{CrGN}$ have serum ANCA. However, some patients with pauci-immune CrGN lack ANCA. Our previous observational study found that about one third of patients with pauci-immune CrGN were ANCA-negative. Compared to their ANCA-positive counterparts, ANCA-negative patients have higher levels of proteinuria, poorer renal outcome and less constitutional and extra-renal symptoms than ANCA-positive patients [25]. Further investigation found that the extent of neutrophil activation 
and degranulation, the pathways of complement activation as well as the prevalence and target antigens of antiendothelial cell antibodies were different between patients with ANCA-negative pauci-immune CrGN and ANCA-positive one [26-29]. These findings suggested that ANCA-negative pauci-immune CrGN represents a distinct disease entity independent of ANCA-positive pauci-immune CrGN.

\section{Management of AAV}

Left untreated, AAV is usually fatal, with a 6-month mortality rate of about $60 \%$ and a 1 -year mortality rate of about $80 \%$ [30]. Since the introduction of immunosuppressive therapy, in particular glucocorticoids in combination with daily oral cyclophosphamide (CTX), the outcome of AAV has dramatically improved. Remission is achieved in about $85 \%$ of patients under such regimens [31]. Today, patients with AAV are treated according to disease stage and activity. On the principle that more severe disease requires more aggressive therapy, the European Vasculitis Study Group (EUVAS) devised a system for subgrouping AAV presentations for different treatment regimens [32]. Plasmapheresis is recommended for patients with severe acute renal failure, diffuse pulmonary hemorrhage or co-existence of antiglomerular basement membrane antibody [31, 33]. In a randomized controlled trial named MEPEX and raised by the EUVAS, in 137 patients who had rapid renal function deterioration and were on dialysis (serum creatinine $>500 \mu \mathrm{mol} / \mathrm{l}$ ) at presentation, compared with intravenous methylprednisolone pulse therapy, plasmapheresis was found to be associated with a higher rate of renal function recovery at 3 months and dialysis-free survival at 12 months [33].

As described above, the cornerstone of treatment for AAV has been the introduction of corticosteroids in combination with daily oral CTX. However, the oral CTX regimen has severe side effects, including secondary infection, bone marrow suppression, hemorrhagic cystitis and bladder carcinoma. The toxicity associated with oral CTX administration has prompted studies of alternative immunosuppressive therapy. Several randomized controlled trials compared the efficacy and safety of oral CTX versus intravenous CTX for induction of remission. Similar rates of remission resulted from these two regimens, and intravenous CTX was associated with a lower cumulative dose and less side effects [34].
Based on the presumed pathogenic potential of ANCA, $B$ cell-targeted therapy has been evaluated. Rituximab was proved to have equivalent efficacy to CTX in initial treatment and did not cause more adverse effects. Rituximab and glucocorticoids are recommended in patients without severe disease or in whom CTX is contraindicated $[35,36]$.

After the induction of remission by CTX, less toxic drugs instead of CTX should be used for maintenance of remission, such as azathioprine, methotrexate, leflunomide or mycophenolate mofetil. Among these medications in maintenance therapy, azathioprine is suggested as the first choice [37]. In 2014, Guillevin et al. [38] reported that their preliminary data indicated that maintenance treatment with rituximab was superior to azathioprine for preventing relapses. However, this finding needs further investigation.

Recently, alternative complement pathway activation has been proved to play an important role in the pathogenesis of human AAV $[39,40]$. In particular C5a, which is generated in complement activation, plays a critical role in ANCA-mediated neutrophil activation [41]. Therefore, inhibition of C5a is a potential therapy approach for AAV. The clinical trial of CCX168, a smallmolecule antagonist of human $\mathrm{C} 5 \mathrm{aR}$, in humans is underway (ClinicalTrials.gov No. NCT01363388).

The treatment for Chinese patients with AAV is consistent with the above recommendations from Western countries. In the early years intravenous CTX was also applied for maintenance of remission [13, 16, 42]. Rituximab in initial treatment is a relatively new treatment regimen in China, and clinical studies on efficacy, side effects and long-term outcomes are still lacking.

\section{Outcomes of AAV in China}

\section{Mortality and Secondary Infection}

As mentioned above, before the introduction of immunosuppressive therapy, the prognosis of AAV was poor. Active vasculitis per se was the most common cause of death at that time. The employment of CTX and corticosteroids substantially improves the prognosis. However, secondary infection substituted active vasculitis to be the leading cause of death thereafter. In a prospective study by the EUVAS, a total of 524 patients with newly diagnosed AAV were recruited and 56 patients died in the first year of follow-up. Therapy-associated adverse events, in particular infection, resulted in many more deaths than active vasculitis per se (28 vs. 8 cases) [43]. In China, our 
recent study included 398 patients with AAV and 135 (33.9\%) died during follow-up, with 83 deaths within the first 12 months after diagnosis. Secondary infection was the leading cause of death (39 cases) during the first year after diagnosis. Secondary infection was also an independent predictor of death. Other independent predictors of all-cause mortality included older age, pulmonary involvement of AAV and initial renal function [44]. In a subgroup study of patients with rapidly progressive glomerulonephritis (RPGN) who were on dialysis at diagnosis, 89 patients were recruited and followed up for 6 months. Infection remained the most common cause of death $(9 / 19,47.4 \%)$, followed by active vasculitis $(5 / 19$, 26.3\%) [45]. This indicated that even in patients with severe ANCA-associated glomerulonephritis, secondary infection, rather than active AAV, is the leading cause of death.

Since secondary infection is a crucial cause of mortality, special efforts were made to investigate the predictors of secondary infection. In the abovementioned study by Lai et al. [44], 39 patients died due to secondary infection within the first year. Further analysis revealed that age, initial renal function, lymphocyte count in the peripheral blood and underlying pulmonary involvement of AAV were found to be independently associated with secondary infection [44]. Our later study revealed that the CD4 lymphocyte count had a higher predictive value than the total lymphocyte count for overall infections [46]. Those findings from the above studies remind physicians to pay special attention to patients at high risk of infection, such as patients with higher age or renal insufficiency or those with pulmonary involvement of AAV. Monitoring the total lymphocyte count, especially the CD4+ lymphocyte count, also becomes increasingly important during immunosuppressive therapy. In some less developed areas where CD4+ lymphocyte count is not available, monitoring total lymphocyte count is also useful since it is correlated closely with the CD4+ lymphocyte count level [46]. Protocols for prevention of infections, such as vaccination and Pneumocystis carinii pneumonia prophylaxis, are helpful to improve the prognosis.

If the AAV patients successfully escaped early mortality, i.e. active vasculitis per se and infection, chronic complications, in particular malignancy and cardiovascular events, would emerge to the surface. Our recent study found that after 12 months from diagnosis, cardiovascular events become the leading cause of death of AAV patients [44]. Therefore, future studies on these issues in AAV patients are of special interest.

\section{Relapse and Treatment Resistance}

Another two important issues of outcomes are relapse of AAV and treatment resistance. When immunosuppression was tapered and eventually stopped, relapses were common in AAV despite initial good control. Relapses were associated with subsequent progression to end-stage renal disease (ESRD) [47]. Several studies were carried out to identify risk factors for relapse and treatment resistance in AAV in Western countries. In two large cohort studies from the USA and France, it was found that PR3-ANCA, lung or upper airway involvement were factors associated with relapse, while age, female, black ethnicity, MPO-ANCA and severe kidney disease might be predictors of treatment resistance [47, 48]. In our recent study which recruited 439 Chinese patients with AAV, treatment resistance occurred in 47 of $439(10.7 \%)$ patients and was independently associated with higher serum creatinine levels. Relapse affected 128 of $392(32.7 \%)$ patients achieving remission and was independently associated with lung involvement and lower serum creatinine level [42]. A study by Chang et al. [49] including 152 patients with AAV found DRB1*0405 to be an independent risk factor of treatment resistance. Another study by Cao et al. [50] which recruited 98 patients with AAV found that female gender and severity of renal disease at presentation were predictors of treatment resistance, and PR3-ANCA and pulmonary involvement were predictors of relapse. The results from the two centers showed some differences, but both studies indicated that patients with severe renal involvement had a lower chance of remission. Since these patients are at higher risk of adverse effects of treatment, especially infection, decisionmaking in treatment protocols needs to take all the above issues into consideration. The mechanism of association between elevated serum creatinine and a lower risk of relapse may be the immunosuppressive state caused by renal insufficiency [51].

Early recognition of relapses is also very crucial in clinical practice. Our previous study found that relapses in AAV were likely to begin with the same organ as in the initial onset [52], which facilitates early recognition of relapses.

Biomarkers which can reflected disease activity or predict relapses are of clinical interest. Neutrophil gelatinase-associated lipocalin (NGAL) is a biomarker of neutrophil degranulation, which is an important aspect in the pathogenesis of AAV. It was found in our study that the levels of serum NGAL closely correlated with the Birmingham Vasculitis Activity Score as well as the level of erythrocyte sedimentation rate, $\mathrm{C}$-reactive protein and 
Table 2. Predictors of different outcomes of AAV in Chinese single-center studies

\begin{tabular}{lll}
\hline Outcome & Predictor & Reference \\
\hline Death & $\begin{array}{l}\text { secondary infection, age, pulmonary involvement, initial renal function } \\
\text { age, pulmonary hemorrhage }\end{array}$ & $\begin{array}{l}\text { Lai et al. [44], 2014 } \\
\text { Li et al. [45], 2013 }\end{array}$ \\
\hline Infection & age, poor renal function, lower lymphocyte counts, pulmonary involvement & Lai et al. [44], 2014 \\
& CD4 lymphocyte count & Shi et al. [46], 2015 \\
\hline Treatment & higher serum creatinine & Li et al. [42], 2014 \\
resistance & DRB1*0405 & Chang et al. [49], 2012 \\
& female, severity of renal disease & Cao et al. [50], 2014 \\
\hline Relapse & lung involvement, lower serum creatinine level & Li et al. [42], 2014 \\
& PR3-ANCA, lung involvement & Cao et al. [50], 2014 \\
\hline ESRD & histopathological classification of Berden et al. [58] & Chang et al. [59], 2012 \\
& DRB1*0405 & Chang et al. [49], 2012 \\
& lower percentages of normal glomeruli, severity of tubular atrophy, interstitial fibrosis ${ }^{1}$ & Li et al. [45], 2013 \\
\hline
\end{tabular}

${ }^{1}$ In patients who had rapid renal function deterioration requiring dialysis.

ANCA [53]. Other biomarkers which could reflect the disease activity of AAV include circulating and urinary levels of high mobility group box-1, circulating levels of anti-plasminogen antibodies and circulating levels of Ddimer [54-57].

\section{Renal Function Restoration and ESRD}

The kidney is one of the most common organs involved in AAV [2]. In 2010, Berden et al. [58] proposed a histopathological classification of ANCA-associated glomerulonephritis, and patients were classified as focal, mixed, crescentic and sclerotic ANCA-associated glomerulonephritis, respectively. During follow-up, the phenotypical order of categories (focal, crescentic, mixed and sclerotic) corresponded to the severity of renal function loss. A study by Chang et al. [59] including 121 Chinese patients with AAV re-evaluated this classification for its prognostic capacity and found this system could predict ESRD, with some difference in sequence compared to the study of Berden et al. The probability of progressing to ESRD increased with ascending categories of focal, mixed, crescentic and sclerotic glomerulonephritis, which is different from Caucasian patients. It was revealed that the histopathological system of Berden et al. could independently predict renal outcome, particularly the development of ESRD, in Chinese patients with AAV.

RPGN is the most severe form of renal lesion in AAV. Renal restoration is a pertinent issue for this subgroup of patients. Our recent study included 89 Chinese patients with ANCA-associated glomerulonephritis who suffered RPGN and were on dialysis at the time of diagnosis. It was found that percentages of normal glomeruli, extent of tubular atrophy and extent of interstitial fibrosis in the renal specimens were predictors of renal function restoration [45].

In our abovementioned study [49] on genetic predictors of outcomes in Chinese patients with AAV, DRB $1 * 0405$ was found to be an independent risk factor of ESRD in AAV patients. However, it is necessary to validate our results in an independent cohort.

Information on predictors for different outcomes of AAV patients may be helpful in treatment decision-making in clinical practice. The main findings of the research cited in this paper are listed in table 2.

\section{Conclusions}

AAV is a multisystem autoimmune diseases and it is not rare in the Chinese population. There is a preponderance of MPA and MPO-ANCA in Chinese patients with AAV. The predictors of various outcomes are, to some extent, different from those from Western countries. 


\section{Acknowledgements}

This study was supported by a grant of the Chinese 973 project (No. 2012CB517702), three grants of the National Natural Science Fund (No. 81425008, No. 81370829 and No. 81321064) and the Research Fund for the Doctoral Program of Higher Education of China (No. 20120001110018).

\section{Disclosure Statement}

The authors declare no conflicts of interest.

\section{References}

$>1$ Jennette JC, Falk RJ, Bacon PA, Basu N, Cid $>_{10}$ MC, Ferrario F, Flores-Suarez LF, Gross WL, Guillevin L, Hagen EC, Hoffman GS, Jayne DR, Kallenberg CG, Lamprecht P, Langford CA, Luqmani RA, Mahr AD, Matteson EL, Merkel PA, Ozen S, Pusey CD, Rasmussen N, Rees AJ, Scott DG, Specks U, Stone JH, Takahashi K, Watts RA: 2012 revised International Chapel Hill Consensus Conference Nomenclature of Vasculitides. Arthritis Rheum 2013;65:1-11.

-2 Jennette JC, Falk RJ: Small-vessel vasculitis. N Engl J Med 1997;337:1512-1523.

-3 Hagen EC, Daha MR, Hermans J, Andrassy K, Csernok E, Gaskin G, Lesavre P, Lüdemann J, Rasmussen N, Sinico RA, Wiik A, van der Woude FJ: Diagnostic value of standardized assays for anti-neutrophil cytoplasmic antibodies in idiopathic systemic vasculitis. EC/ BCR Project for ANCA Assay Standardization. Kidney Int 1998;53:743-753.

4 Finkielman JD, Merkel PA, Schroeder D, Hoffman GS, Spiera R, St Clair EW, Davis JC Jr, McCune WJ, Lears AK, Ytterberg SR, Hummel AM, Viss MA, Peikert T, Stone JH, Specks U: Antiproteinase 3 antineutrophil cytoplasmic antibodies and disease activity in Wegener granulomatosis. Ann Intern Med 2007;147:611-619.

5 Watts RA, Lane SE, Scott DG, Koldingsnes W, Nossent H, Gonzalez-Gay MA, GarciaPorrua C, Bentham GA: Epidemiology of vasculitis in Europe. Ann Rheum Dis 2001;60 1156-1157.

6 Watts RA, Scott DG: L32. ANCA vasculitis over the world. What do we learn from country differences? Presse Med 2013;42:591-593.

7 Tervaert JW, Stegeman CA, Kallenberg CG: Silicon exposure and vasculitis. Curr Opin Rheumatol 1998;10:12-17.

8 Chen M, Yu F, Zhang Y, Zhao MH: Clinical and pathological characteristics of Chinese patients with antineutrophil cytoplasmic autoantibody associated systemic vasculitis: a study of 426 patients from a single center. Postgrad Med J 2005;81:723-727.

-9 Shuai ZW, Lv YF, Zhang MM, Hu ZY: Clinical analysis of patients with myeloperoxidase antineutrophil cytoplasmic antibody-associated vasculitis. Genet Mol Res 2015;14:52965303.
Chen M, Cui Z, Zhao MH: ANCA-associated vasculitis and anti-GBM disease: the experience in China. Nephrol Dial Transplant 2010; 25:2062-2065.

11 Zhu P, Zhou FD, Zhao MH: The renal histopathology spectrum of elderly patients with kidney diseases: a study of 430 patients in a single Chinese center. Medicine (Baltimore) 2014;93:e226.

12 Liu LJ, Chen M, Yu F, Zhao MH, Wang HY: Evaluation of a new algorithm in classification of systemic vasculitis. Rheumatology (Oxford) 2008;47:708-712.

13 Chen M, Yu F, Zhang Y, Zou WZ, Zhao MH, Wang HY: Characteristics of Chinese patients with Wegener's granulomatosis with antimyeloperoxidase autoantibodies. Kidney Int 2005;68:2225-2229.

14 Chen M, Yu F, Wang SX, Zou WZ, Zhang Y, Zhao MH, Wang HY: Renal histology in Chinese patients with anti-myeloperoxidase autoantibody-positive Wegener's granulomatosis. Nephrol Dial Transplant 2007;22:139-145.

15 Liu LJ, Chen M, Yu F, Zhao MH, Wang HY: IgG subclass distribution, affinity of anti-myeloperoxidase antibodies in sera from patients with Wegener's granulomatosis and microscopic polyangiitis. Nephrology (Carlton) 2008;13:629-635.

16 Chen M, Yu F, Zhang Y, Zhao MH: Antineutrophil cytoplasmic autoantibody-associated vasculitis in older patients. Medicine (Baltimore) 2008;87:203-209.

$\checkmark 17$ Luo H, Chen M, Yang R, Xu PC, Zhao MH: The association of HLA-DRB1 alleles with antineutrophil cytoplasmic antibody-associated systemic vasculitis in Chinese patients. Hum Immunol 2011;72:422-425.

18 Wu Z, Wu Q, Xu J, Chen S, Sun F, Li P, Bai Y, Zheng W, Chen H, Zhang F, Li Y: HLA-DPB1 variant rs3117242 is considered to be associated with GPA in a Han Chinese population. Int J Rheum Dis 2015, Epub ahead of print.

19 Gao Y, Zhao MH, Guo XH, Xin G, Gao Y, Wang HY: The prevalence and target antigens of antithyroid drugs induced antineutrophil cytoplasmic antibodies (ANCA) in Chinese patients with hyperthyroidism. Endocr Res 2004;30:205-213.

20 Chen M, Gao Y, Guo XH, Zhao MH: Propylthiouracil-induced antineutrophil cytoplasmic antibody-associated vasculitis. Nat Rev Nephrol 2012;8:476-483.
21 Gao Y, Chen M, Ye H, Guo XH, Zhao MH, Wang HY: The target antigens of antineutrophil cytoplasmic antibodies (ANCA) induced by propylthiouracil. Int Immunopharmacol 2007; 7:55-60.

22 Zhao MH, Chen M, Gao Y, Wang HY: Propylthiouracil-induced anti-neutrophil cytoplasmic antibody-associated vasculitis. Kidney Int 2006;69:1477-1481.

23 Yu F, Chen M, Gao Y, Wang SX, Zou WZ, Zhao MH, Wang HY: Clinical and pathological features of renal involvement in propylthiouracil-associated ANCA-positive vasculitis. Am J Kidney Dis 2007;49:607-614.

24 Gao Y, Ye H, Yu F, Guo XH, Zhao MH: Antimyeloperoxidase IgG subclass distribution and avidity in sera from patients with propylthiouracil-induced antineutrophil cytoplasmic antibodies associated vasculitis. Clin Immunol 2005;117:87-93.

25 Chen M, Yu F, Wang SX, Zou WZ, Zhao MH, Wang HY: Antineutrophil cytoplasmic autoantibody-negative pauci-immune crescentic glomerulonephritis. J Am Soc Nephrol 2007; 18:599-605.

26 Wang F, Chen M, Zhao MH: Neutrophil degranulation in antineutrophil cytoplasmic antibody-negative pauci-immune crescentic glomerulonephritis. J Nephrol 2009;22:491496.

27 Xing GQ, Chen M, Liu G, Wang SX, Zhao $\mathrm{MH}$ : Renal neutrophils infiltration in antineutrophil cytoplasmic antibodies-negative pauci-immune crescentic glomerulonephritis. Am J Med Sci 2010;340:474-480.

28 Cong M, Chen M, Zhang JJ, Hu Z, Zhao MH Anti-endothelial cell antibodies in antineutrophil cytoplasmic antibodies negative pauci-immune crescentic glomerulonephritis. Nephrology (Carlton) 2008;13:228-234.

29 Xing GQ, Chen M, Liu G, Zheng X, E J, Zhao $\mathrm{MH}$ : Differential deposition of C4d and MBL in glomeruli of patients with ANCA-negative pauci-immune crescentic glomerulonephritis. J Clin Immunol 2010;30:144-156.

30 Booth AD, Almond MK, Burns A, Ellis P, Gaskin G, Neild GH, Plaisance M, Pusey CD, Jayne DR: Outcome of ANCA-associated renal vasculitis: a 5-year retrospective study. Am J Kidney Dis 2003;41:776-784. 
31 Kidney Disease: Improving Global Outcomes (KDIGO) Glomerulonephritis Work Group: KDIGO clinical practice guideline for glomerulonephritis. Kidney Int Suppl 2012;2: 139-274.

32 Jayne DR, Rasmussen N: Treatment of antineutrophil cytoplasm autoantibody-associated systemic vasculitis: initiatives of the European Community Systemic Vasculitis Clinical Trials Study Group. Mayo Clin Proc 1997;72: 737-747.

-33 Jayne DR, Gaskin G, Rasmussen N, Abramowicz D, Ferrario F, Guillevin L, Mirapeix E, Savage CO, Sinico RA, Stegeman CA, Westman KW, van der Woude FJ, de Lind van Wijngaarden RA, Pusey CD: Randomized trial of plasma exchange or high-dosage methylprednisolone as adjunctive therapy for severe renal vasculitis. J Am Soc Nephrol 2007;18: 2180-2188.

34 de Groot K, Harper L, Jayne DR, Flores Suarez LF, Gregorini G, Gross WL, Luqmani R, Pusey CD, Rasmussen N, Sinico RA, Tesar V, Vanhille P, Westman K, Savage CO: Pulse versus daily oral cyclophosphamide for induction of remission in antineutrophil cytoplasmic antibody-associated vasculitis: a randomized trial. Ann Intern Med 2009;150:670-680.

- 35 Stone JH, Merkel PA, Spiera R, Seo P, Langford CA, Hoffman GS, Kallenberg CG, St Clair EW, Turkiewicz A, Tchao NK, Webber L, Ding L, Sejismundo LP, Mieras K, Weitzenkamp D, Ikle D, Seyfert-Margolis V, Mueller M, Brunetta P, Allen NB, Fervenza FC, Geetha D, Keogh KA, Kissin EY, Monach PA, Peikert T, Stegeman C, Ytterberg SR, Specks U: Rituximab versus cyclophosphamide for ANCAassociated vasculitis. N Engl J Med 2010;363: 221-232.

36 Jones RB, Tervaert JW, Hauser T, Luqmani R, Morgan MD, Peh CA, Savage CO, Segelmark M, Tesar V, van Paassen P, Walsh D, Walsh $M$, Westman K, Jayne DR: Rituximab versus cyclophosphamide in ANCA-associated renal vasculitis. N Engl J Med 2010;363:211220.

- 37 Jayne D, Rasmussen N, Andrassy K, Bacon P, Tervaert JW, Dadoniené J, Ekstrand A, Gaskin G, Gregorini G, de Groot K, Gross W, Hagen EC, Mirapeix E, Pettersson E, Siegert C, Sinico A, Tesar V, Westman K, Pusey C: A randomized trial of maintenance therapy for vasculitis associated with antineutrophil cytoplasmic autoantibodies. N Engl J Med 2003; 349:36-44.

-38 Guillevin L, Pagnoux C, Karras A, Khouatra C, Aumaitre O, Cohen P, Decaux O, Desmurs-Clavel H, Gobert P, Quemeneur T, Blanchard-Delaunay C, Godmer P, Puechal X, Carron PL, Hatron PY, Limal N, Hamidou
M, Bonnotte B, Ravaud P, Mouthon L; French Vasculitis Study Group: Rituximab versus azathioprine for maintenance in ANCA-associated vasculitis. N Engl J Med 2014;371: 1771-1780.

39 Xing GQ, Chen M, Liu G, Heeringa P, Zhang JJ, Zheng X, E J, Kallenberg CG, Zhao MH; Complement activation is involved in renal damage in human antineutrophil cytoplasmic autoantibody associated pauci-immune vasculitis. J Clin Immunol 2009;29:282-291.

40 Gou SJ, Yuan J, Chen M, Yu F, Zhao MH: Circulating complement activation in patients with anti-neutrophil cytoplasmic antibodyassociated vasculitis. Kidney Int 2013;83:129137.

41 Schreiber A, Xiao H, Jennette JC, Schneider W, Luft FC, Kettritz R: C5a receptor mediates neutrophil activation and ANCA-induced glomerulonephritis. J Am Soc Nephrol 2009; 20:289-298.

42 Li ZY, Chang DY, Zhao MH, Chen M: Predictors of treatment resistance and relapse in antineutrophil cytoplasmic antibody-associated vasculitis: a study of 439 cases in a single Chinese center. Arthritis Rheumatol 2014;66: 1920-1926.

43 Little MA, Nightingale P, Verburgh CA, Hauser T, De Groot K, Savage C, Jayne D, Harper L: Early mortality in systemic vasculitis: relative contribution of adverse events and active vasculitis. Ann Rheum Dis 2010; 69:1036-1043.

44 Lai QY, Ma TT, Li ZY, Chang DY, Zhao MH, Chen M: Predictors for mortality in patients with antineutrophil cytoplasmic autoantibodyassociated vasculitis: a study of 398 Chinese patients. J Rheumatol 2014;41:1849-1855.

-45 Li ZY, Gou SJ, Chen M, Zhao MH: Predictors for outcomes in patients with severe ANCAassociated glomerulonephritis who were dialysis-dependent at presentation: a study of 89 cases in a single Chinese center. Semin Arthritis Rheum 2013;42:515-521.

46 Shi YY, Li ZY, Zhao MH, Chen M: The CD4 lymphocyte count is a better predictor of overall infection than the total lymphocyte count in ANCA-associated vasculitis under a corticosteroid and cyclophosphamide regimen: a retrospective cohort. Medicine (Baltimore) 2015;94:e843.

47 Hogan SL, Falk RJ, Chin H, Cai J, Jennette CE, Jennette JC, Nachman PH: Predictors of relapse and treatment resistance in antineutrophil cytoplasmic antibody-associated smallvessel vasculitis. Ann Intern Med 2005;143: 621-631.

48 Pagnoux C, Hogan SL, Chin H, Jennette JC, Falk RJ, Guillevin L, Nachman PH: Predictors of treatment resistance and relapse in anti- neutrophil cytoplasmic antibody-associated small-vessel vasculitis: comparison of two independent cohorts. Arthritis Rheum 2008;58: 2908-2918.

49 Chang DY, Luo H, Zhou XJ, Chen M, Zhao $\mathrm{MH}$ : Association of HLA genes with clinical outcomes of ANCA-associated vasculitis. Clin J Am Soc Nephrol 2012;7:1293-1299.

50 Cao Y, Tian Z, Li W, Ma L, Yu Y, Ren W: Predictors of treatment resistance and relapse in Chinese patients with antineutrophil cytoplasmic antibody-associated disease. J Rheumatol 2014;41:916-922.

51 Cohen G, Rudnicki M, Hörl WH: Uremic toxins modulate the spontaneous apoptotic cell death and essential functions of neutrophils. Kidney Int Suppl 2001;78:S48-S52.

52 Chen M, Yu F, Zhao MH: Relapses in patients with antineutrophil cytoplasmic autoantibody-associated vasculitis: likely to begin with the same organ as initial onset. J Rheumatol 2008;35:448-450.

53 Chen M, Wang F, Zhao MH: Circulating neutrophil gelatinase-associated lipocalin: a useful biomarker for assessing disease activity of ANCA-associated vasculitis. Rheumatology (Oxford) 2009;48:355-358.

54 Wang C, Gou SJ, Chang DY, Yu F, Zhao MH, Chen M: Association of circulating level of high mobility group box 1 with disease activity in antineutrophil cytoplasmic autoantibody-associated vasculitis. Arthritis Care Res (Hoboken) 2013;65:1828-1834.

$55 \mathrm{Ma}$ TT, Wang H, Wang C, Chang DY, Zhao $\mathrm{MH}$, Chen M: Urinary levels of high mobility group box-1 are associated with disease activity in antineutrophil cytoplasmic autoantibody-associated vasculitis. PLoS One 2015. 10:e0123586.

56 Hao J, Wang C, Gou SJ, Zhao MH, Chen M: The association between anti-plasminogen antibodies and disease activity in ANCA-associated vasculitis. Rheumatology (Oxford) 2014;53:300-306.

57 Ma TT, Huang YM, Wang C, Zhao MH, Chen M: Coagulation and fibrinolysis index profile in patients with ANCA-associated vasculitis. PLoS One 2014;9:e97843.

58 Berden AE, Ferrario F, Hagen EC, Jayne DR, Jennette JC, Joh K, Neumann I, Noël LH, Pusey CD, Waldherr R, Bruijn JA, Bajema IM: Histopathologic classification of ANCAassociated glomerulonephritis. J Am Soc Nephrol 2010;21:1628-1636.

59 Chang DY, Wu LH, Liu G, Chen M, Kallenberg CG, Zhao MH: Re-evaluation of the histopathologic classification of ANCA-associated glomerulonephritis: a study of 121 patients in a single center. Nephrol Dial Transplant 2012;27:2343-2349. 